\title{
Dust Properties of Super-Nova Remnant (Crab Nebula) Using AKARI Survey
}

\section{A. K. Jha, A. Yadav, D. R. Upadhyay and B. Aryal}

\section{Journal of Nepal Physical Society}

Volume 7, Issue 4, December 2021

ISSN: 2392-473X (Print), 2738-9537 (Online)

\section{Editors:}

Dr. Binod Adhikari

Dr. Bhawani Joshi

Dr. Manoj Kumar Yadav

Dr. Krishna Rai

Dr. Rajendra Prasad Adhikari

Mr. Kiran Pudasainee

JNPS, 7 (4), 64-70 (2021)

DOI: http://doi.org/10.3126/jnphyssoc.v7i4.42933

$$
\text { JNPS }
$$

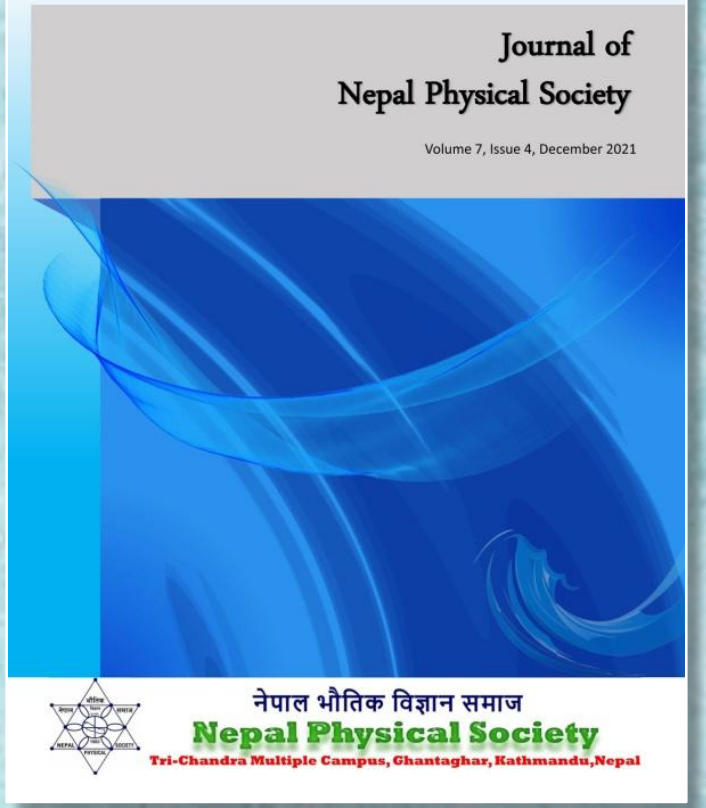

Published by:

Nepal Physical Society

P.O. Box: 2934

Tri-Chandra Campus

Kathmandu, Nepal

Email:nps.editor@gmail.com 


\title{
Dust Properties of Super-Nova Remnant (Crab Nebula) Using AKARI Survey
}

\author{
A. K. Jha ${ }^{1}$, A. $\operatorname{Yadav}^{1}$, D. R. Upadhyay ${ }^{2, ~ *}$ and B. Aryal ${ }^{1}$ \\ ${ }^{1}$ Central Department of Physics, Tribhuvan University, Nepal \\ ${ }^{2}$ Department of Physics, Amrit Campus, Tribhuvan University, Nepal \\ *Corresponding Email: mnadphy03@gmail.com/devendra.upadhyay@ac.tu.edu.np
}

Received: 12th November, 2021; Revised: 10th December, 2021; Accepted: 27th December, 2021

\begin{abstract}
We present the distribution of dust color temperature, Planck's function, dust mass, and visual extinction in the far-infrared $(140 \mu \mathrm{m}$ and $90 \mu \mathrm{m})$ AKARI surveys of the Crab Nebula using Sky View Virtual Observatory. With a systematic search of a supernova remnants, we found Crab Nebula at R. A. (J2000) / Decl. (J2000) $=05^{\mathrm{h}} 34^{\prime} 31.94 " /+22^{0} 00^{\prime} 52.20^{\prime \prime}$. The maximum flux is found to be $145.94 \mathrm{MJysr}^{-1}$ at $140 \mu \mathrm{m}$ and $177.01 \mathrm{MJysr}^{-1}$ at $90 \mu \mathrm{m}$ wavelength. The total dust mass of the Supernova remnant is estimated to be $6.11 \times 10^{39} \mathrm{~kg}\left(3.07 \times 10^{9} \mathrm{M}_{\odot}\right)$ and the dust color temperature is found in the range between $31.16 \mathrm{~K}$ to $47.11 \mathrm{~K}$ with mean value of temperature of $38.07 \mathrm{~K}$. Similarly we obtained the value of Planck's function in the range of $3.96 \times 10^{-15} \mathrm{Wm}^{-2} \mathrm{sr}^{-1} \mathrm{~Hz}^{-1}$ to $1.96 \times 10^{-14} \mathrm{Wm}^{-2} \mathrm{sr}^{-1} \mathrm{~Hz}^{-1}$ with average value $9.59 \times 10^{-15} \mathrm{Wm}^{-2} \mathrm{sr}^{-}$ ${ }^{1} \mathrm{~Hz}^{-1}$. The value of visual extinction ranges from $9.21 \times 10^{-14}$ mag to $2.70 \times 10^{-13}$ mag with mean value of $1.57 \times 10^{-13} \mathrm{mag}$. The distribution trend of flux, dust color temperature, and Planck's function are nearly similar, whereas dust mass and visual extinction follow an inverse relationship with the previous three parameters.
\end{abstract}

Keywords: AKARI; Crab Nebula; Dust Color Temperature; Dust mass; Visual Extinction.

\section{INTRODUCTION}

A supernova is the result of a star ending by a powerful explosion leaving behind an expanding gaseous remnant. The origin of interstellar medium, black holes, X-ray binaries, etc. are connected to super-nova explosion [1-3]. Supernovae is the main cause of efficient dust in the galaxies. Using $100 \mu \mathrm{m}$ and $60 \mu \mathrm{m}$ IRAS maps, Kiss et al. (2004) and Koenyves et al. (2007) investigated 462 far-infrared loops, studied their luminosity distributions and concluded that these structures are formed and governed by supernovae and young stellar winds at the low Galactic latitude[4, 5]. The dust in the universe is mostly from the explosion that may be from star formation or star explosion or maybe a collision between meteoroids, maybe others things which can't be discovered yet [6-9]. Matsuura et al. had also studied that the dust in the universe is from SN eject [10]. Dust plays an important role in our earth, our solar system and also the whole universe. The radiation in the galaxies in the space is also due to the dust evolved from the supernova explosion [11]. Matsuura et al., (2015) confirmed large mass of dust in the eject of supernova $\mathrm{SNe}$ $1987 \mathrm{~A}$ is about 0.4 to $0.7 \mathrm{M}_{\odot}$ and according to them core collapse supernova (CCSNe) is the main source of interstellar dust [12]. Supernova remnants (SNRs) play an important key role in understanding supernovae explosion mechanism, star formation, the phenomenon of dust formation mechanism, exploring the likely sources of Galactic cosmic rays, and the chemical enrichment of interstellar medium (ISM) [13-15]. Supernova remnants are very important because it gives the structure of the galaxies. They are also the source of heating of the interstellar gas by the means of magnetic turbulence and violent shocks. It is important for our evolution because it forms the heaviest elements like iron, magnesium, copper, gold, uranium etc. The Milky Way Galaxy contains over 200 supernova remnants which 
make our galaxy beautiful. It is the source of energy and heavy elements [13-15]. Green et al. (2004) suggest that $77 \%$ of the remnants are closed as shell, $12 \%$ are composite and remaining and $4 \%$ have not observed clearly [16]. These facts suggest that interstellar dust plays an important role in shaping mechanism of ISM. Several authors studied about dust environment around Nebula, Pulsars, AGB stars and white dwarfs using IRAS, IRIS AKARI band and survey they found two digit and very low kelvin dust color temperature [17-26]. So, we studied the physical properties interstellar dust like dust temperature, distribution of Planck's function, dust mass, and visual extinction, etc. near the supernova remnant crab nebula.

\section{METHODS AND MATERIALS}

\subsection{Database}

We used SkyView virtual Observatory (https://skyview.gsfc.nasa.gov) in order to search an isolated nebular structure at different AKARI wavelength bands nearby the supernova remnant i.e., Crab Nebula for our observation of dust abundance. We followed the method used by Jha and Aryal, (2017) during the search of best candidate $[24,26]$.

We found an isolated nebular structure at R. A. (J2000): $05^{\mathrm{h}} 34^{\prime}$ 940", Decl. (J2000): +22 ${ }^{\mathrm{o}} 00^{\prime}$ 52.20 , and downloaded the FITS images in two different bands of AKARI $(90 \mu \mathrm{m}$ and $140 \mu \mathrm{m})$ for our datasets.
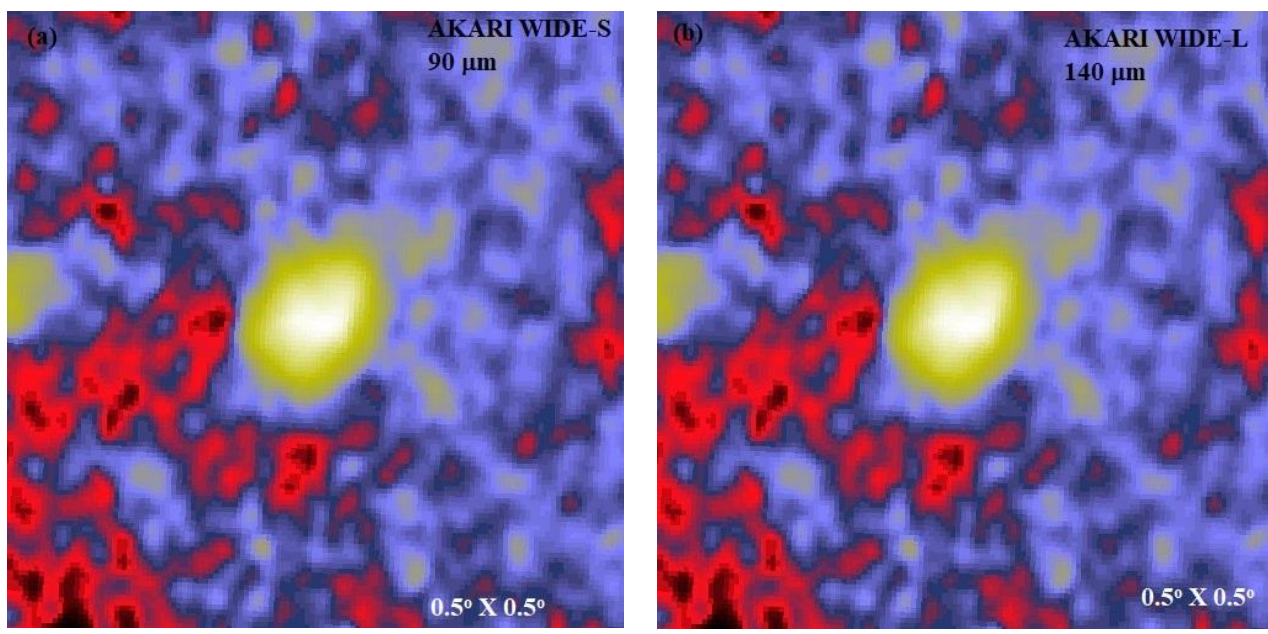

Fig. 1: The image size(degree) of $0.5^{\circ} \times 0.5^{\circ}$ AKARI images of nebular structure in $90 \mu \mathrm{m}$, and $140 \mu \mathrm{m}$ bands with R.A./Decl.(J2000) $=05^{\mathrm{h}} 34^{\prime} 31.940^{\prime \prime} /+22^{0} 00^{\prime} 52.20^{\prime \prime}($ SkyView Query Form (nasa.gov) ).

The FITS images were downloaded and processed using software Aladin v11.0.

\subsection{Dust Color Temperature}

For the estimation of dust color temperature. The flux density emitted at a wavelength $\lambda_{\mathrm{i}}$ is given by [27-29]

$$
F=\left[\frac{2 h c}{\lambda_{i}^{3}\left(e^{\frac{h c}{\lambda_{i} K T_{d}}-1}\right)}\right] N_{d} \alpha \lambda_{i}^{-\beta} \Omega_{i}
$$

where, $\mathrm{N}_{\mathrm{d}}$ is the dust grains column density, $\alpha$ is a constant which depend on the flux and optical depth of the dust, $\beta$ is emissivity index varies with wavelength, $\lambda_{i}$ and $\Omega_{i}$ is the solid angle made by the detector.

To obtain the dust color temperature equations let us consider $T_{\mathrm{d}} \ll<1$ and $\Omega_{90}=\Omega_{140}$ and also $\lambda_{140}=$ $\frac{h c}{k T_{140}}$ and $\lambda_{90}=\frac{h c}{k T_{90}}$, the ratio of flux densities at 90 $\mu \mathrm{m}, \mathrm{F}(90 \mu \mathrm{m})$ and $140 \mu \mathrm{m}$,

$\mathrm{F}(140 \mu \mathrm{m})$ gives the value of $\mathrm{R}$ as:

$R=\left[\frac{\left(e^{\frac{h c}{\lambda_{140} K T} d}-1\right)}{\left(e^{\frac{h c}{\lambda_{90} K T}}-1\right)}\right] 0.64^{-(3+\beta)}$

By substituting the value of $T_{140}=103 \mathrm{~K}$ and $T_{90}=$ $160 \mathrm{~K}$ in equation (2)

$R=\left[\frac{\left(e^{\frac{103}{T_{d}}}-1\right)}{\left(e^{\frac{160}{T_{d}}}-1\right)}\right] 0.64^{-(3+\beta)}$

The value of $\beta$ depends upon the properties of dust grains such as size, composition, compactness etc. 
For the pure black body $\beta=0$, for amorphous layerlattice mater $\beta=1$ and for metal and crystalline dielectrics $\beta=2$. For smaller value of $T_{\mathrm{d}}, 1$ can be dropped from both numerator and denominator of above equation (3) and it takes the form

$$
R=0.64^{-(3+\beta)}\left[\frac{\left(e^{\frac{103}{T} d}\right)}{\left(e^{\frac{160}{T_{d}}}\right)}\right]
$$

Taking natural logarithm on both sides of equation (4) we get

$$
\ln (R)=\ln \left(0.64^{-(3+\beta)}\right)\left[\frac{\frac{103}{T_{d}}}{\frac{160}{T_{d}}}\right]
$$

$T_{\mathrm{d}}=\frac{-57}{\ln \left[R \times 0.64^{(3+\beta)}\right]}$

Which is the required Dust color temperature of the supernova remnant using AKARI 90 and $140 \mu \mathrm{m}$ wavelength.

Where, $R=\frac{F(90)}{F(140)}$

Equation (6) is used for the determination of the dust grain temperature in the remnant of the Supernova $[24,26]$.

\subsection{Dust Mass Estimation}

Dust mass can be estimated by the method of Hilderbrand (1983) and Young et al. (1983). According to them the dust mass can be calculated from infrared flux densities [30, 31] as:

$M_{d u s t}=\frac{4}{3} \frac{a \rho}{Q_{v}}\left[\frac{S_{v} D^{2}}{B\left(v, T_{d}\right)}\right]$

where,

$a=$ weighted grain size

$\rho=$ grain density

$D=$ Distance of the Structure

$\mathrm{Q}_{\mathrm{v}}=$ grain emissivity

$\mathrm{S}_{v}=f \times 5.288 \times 10^{-9} \mathrm{MJy} \mathrm{sr}^{-1}$

For $140 \mu \mathrm{m}$ emitter

$a=0.1 \mu \mathrm{m}$

$\rho=$ grain density $=3000 \mathrm{~kg} \mathrm{~m}^{-3}$,

$\mathrm{Q}_{\mathrm{v}}=$ grain emissivity $=0.0010($ for $140 \mu \mathrm{m})$

$\mathrm{Q}_{\mathrm{v}}=$ grain emissivity $=0.0046($ for $90 \mu \mathrm{m})$

Where, $1 \mathrm{MJysr}^{-1}=1.26 \times 10^{-19} \mathrm{kgs}^{-2}$

and $f=$ relative flux density measured from the Groningen AKARI.
For $140 \mu \mathrm{m}$ wavelength, the expression for the dust mass reduces to,

$$
M_{\text {dust }}=0.40\left[\frac{S_{v} D^{2}}{B\left(v, T_{d}\right)}\right]
$$

The value of Planck's function $B\left(v, T_{\mathrm{d}}\right)$ varies with frequency, and temperature. The Planck's function is a well-known function, given by

$B(v, T)=\frac{2 h v^{3}}{c^{2}}\left[\frac{1}{\exp \left(\frac{h v}{k T_{d}}\right)-1}\right]$

where,

$\mathrm{h}=$ Planck's constant

$\mathrm{c}=$ velocity of light

$v=$ frequency at which the emission is observed

$T_{\mathrm{d}}=$ Dust color temperature of each pixel

\subsection{Visual Extinction}

For estimation of visual extinction optical depth can be written as $[26,27]$

$\tau_{140}=\left(\frac{F_{140 \mu m}}{B\left(\nu, T_{d}\right)_{140}}\right)$

Here $\mathrm{F}_{140 \mu \mathrm{m}}$ in $\mathrm{kgs}^{-2}$ and $\mathrm{B}\left(v, T_{\mathrm{d}}\right)_{140}$ in $\mathrm{Wm}^{-2} \mathrm{sr}^{-1} \mathrm{~Hz}^{-1}$ at $140 \mu \mathrm{m}$ flux in AKARI as suggested by Wood et al. [26] for IRAS survey. For IRIS and AKARI survey, following empirical equation is used to estimate the visual extinction $[26,27]$

$A_{v}=15.078\left(1-e^{\frac{-\tau_{140}}{641.3}}\right)$

\section{RESULTS AND DISCUSSION \\ 3.1 Projection Map}

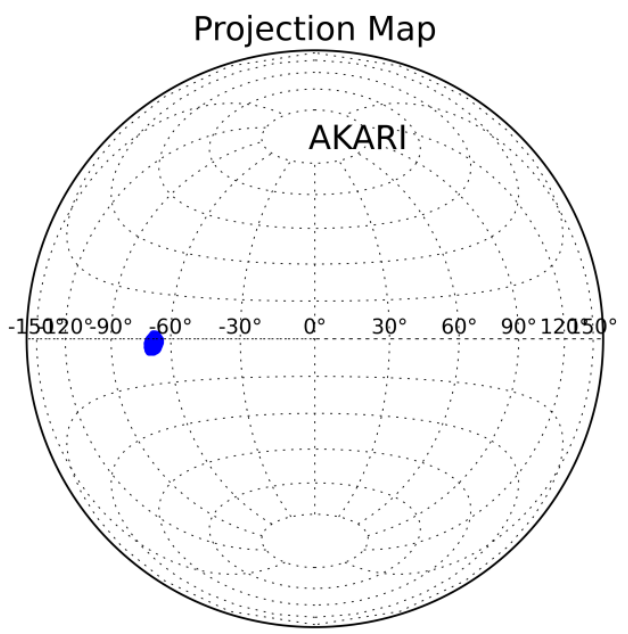

Fig. 2: The projection map of our candidate. 
The selected nebular structure centered at R. A. $(\mathrm{J} 2000) /$ Decl. $(\mathrm{J} 2000)=05^{\mathrm{h}} 34^{\prime} 31.94 \mathrm{\prime} /+22^{0} 00^{\prime}$ 52.20 " is shown in projection map with blue color in Figure 2. From this map it is seen that our structure lies near to the equatorial region.

Figure 3 shows the plot of $90 \mu \mathrm{m}$ flux versus $140 \mu \mathrm{m}$ AKARI flux density scattered plot. The equation of best fitted line is given by,

$F(90)=1.28 F(140)-44.63$

with the slope of value 1.28 and correlation coefficient $R^{2}=0.94$. The slope 1.28 explains that for every additional unit $\mathrm{MJysr}^{-1}$ in flux density at $140 \mu \mathrm{m}$ the flux density at $90 \mu \mathrm{m}$ increase by an average of $1.28 \mathrm{MJysr}^{-1}$. This shows the strongest linear relationship occurred between these two parameters.

\subsection{Contour maps}

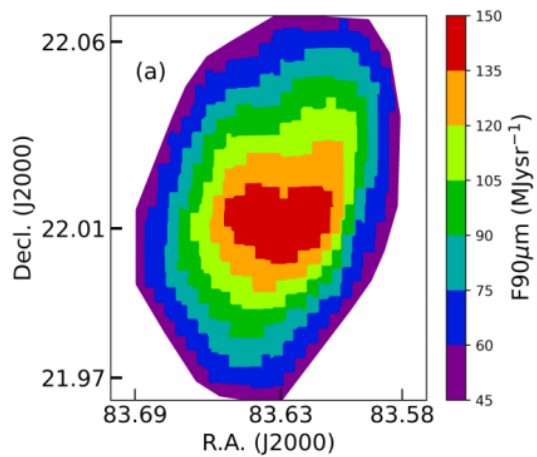

Fig. 4: The contour maps of (a) $\mathrm{F}(90 \mu \mathrm{m})$ and (b) $\mathrm{F}(140 \mu \mathrm{m})$ along with their color scale.

Figure 4(a), (b) shows the contour map of the flux density at $90 \mu \mathrm{m}$ and $140 \mu \mathrm{m} \mathrm{MJysr}^{-1}$ For these contour plot we had plotted the R.A. (J2000) in Xaxis and Decl. (J2000) in y-axis and flux as color map. Figure 4(a), (b) represents a two-dimensional contour plot with the projection of fluxes in the XY

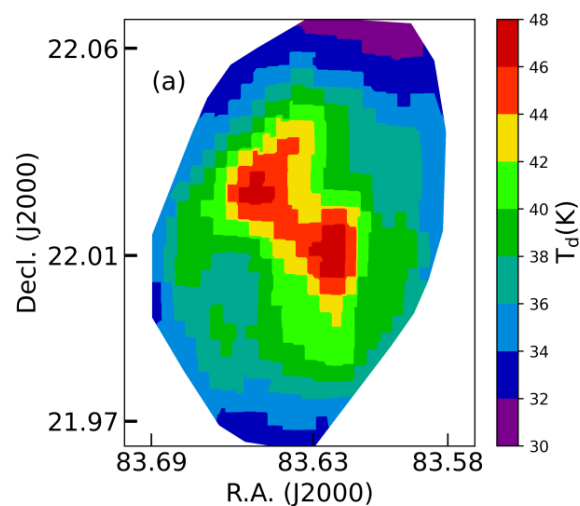

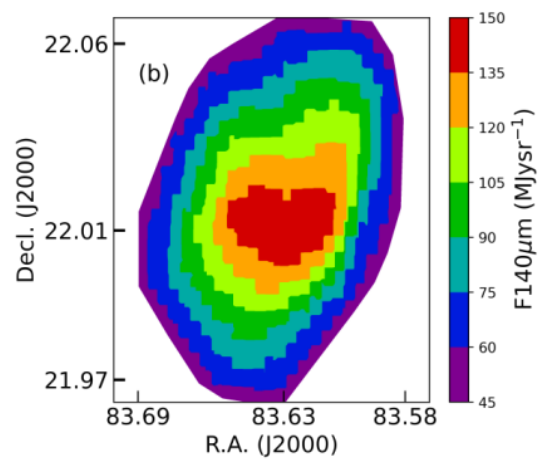

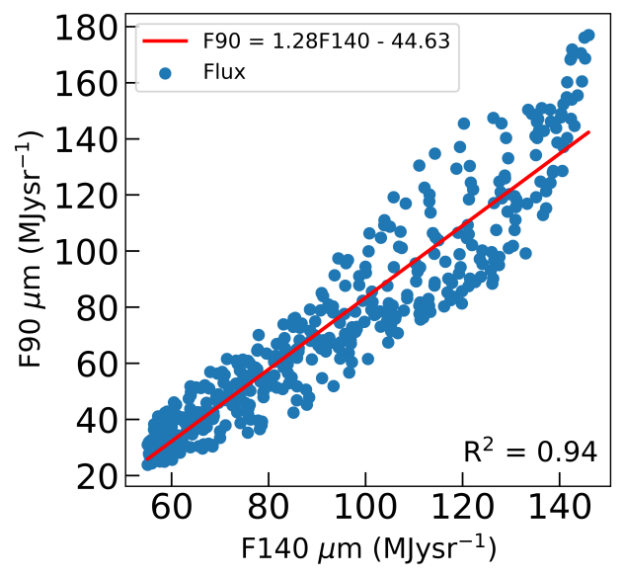

Fig. 3: Correlation between relative flux density at 90 $\mu \mathrm{m}$ AKARI $F(90)$, and flux density at $140 \mu \mathrm{m}$ AKARI $F(140)$ of the structure. The solid line represents the best fit line of the scatter plot.

plane. We obtained that the range of flux density at $90 \mu \mathrm{m} 23.89 \mathrm{MJysr}^{-1}$ to $177.01 \mathrm{MJysr}^{-1}$ with average $72.55 \mathrm{MJysr}^{-1}$ where as fo4 $140 \mu \mathrm{m}$ band 55.01 $\mathrm{MJysr}^{-1}$ to $145.94 \mathrm{MJysr}^{-1}$ with average 91.47 $\mathrm{MJysr}^{-1}$. It indicates that there is more deviation in case of $90 \mu \mathrm{m}$ emission.

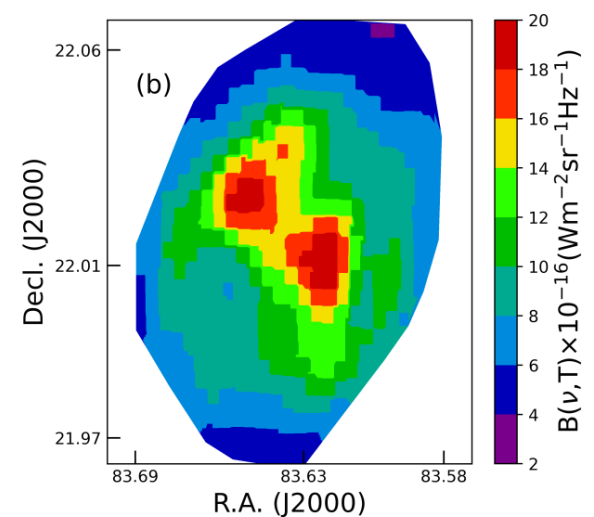

Fig. 5: Contour maps of (a) dust color temperature and (b) Planck's function along with their color scale. 
Figure 5 (a) and (b) shows the contour map of the dust color temperature $\left(T_{d}\right)$ in Kelvin and Planck's function $\left(\mathrm{B}\left(\mathrm{v}, T_{\mathrm{d}}\right)\right.$ in $\mathrm{Wm}^{-2} \mathrm{sr}^{-1} \mathrm{~Hz}^{-1}$.

For these contour plot we had plotted the R.A. (J2000) in X-axis and Decl. (J2000) in Y-axis and dust color temperature and Planck's function as third parameter along with color bar. In figure violet to red indicates increasing sequence of physical parameters. In this case we obtained the range of dust color temperature from $31.16 \mathrm{~K}$ to $47.11 \mathrm{~K}$ and whose,

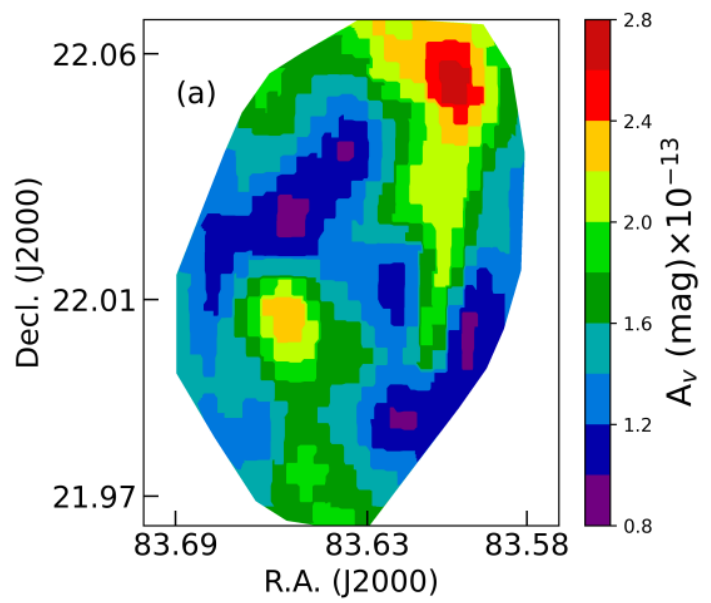

average value $38.07 \mathrm{~K}$. Similarly values of Planck's function varies from $3.96 \times 10^{-15} \mathrm{Wm}^{-2} \mathrm{sr}^{-1} \mathrm{~Hz}^{-1}$ to $1.96 \times 10^{-14} \mathrm{Wm}^{-2} \mathrm{sr}^{-1} \mathrm{~Hz}^{-1}$ and its mean value found to be $9.59 \times 10^{-15} \mathrm{Wm}^{-2} \mathrm{sr}^{-1} \mathrm{~Hz}^{-1}$. The central part of the structure was found to be hotter than outer region as a consequences the Planck's function higher at the core region than outer region.

Figure 6(a) and (b) shows the contour map of the visual extinction $\left(A_{v}\right)$ in mag and dust mass $\left(M_{\mathrm{d}}\right)$ in kilogram.

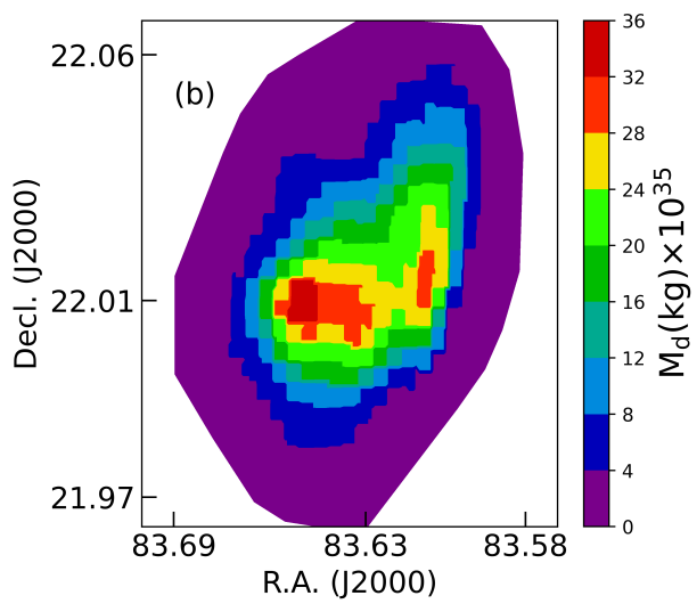

Fig. 6: Contour maps of (a) visual extinction and (b) dust mass along with their color scale.

For these contour plot we had plotted the R.A. (J2000) in X-axis and Decl. (J2000) in Y-axis and visual extinction and dust mass in as color map.

In figure violet to red indicates increasing sequence of physical quantity values. In this case we obtained the range of visual extinction from $9.21 \times 10^{-14} \mathrm{mag}$ to $2.70 \times 10^{-13} \mathrm{mag}$ and average value $1.57 \times 10^{-13} \mathrm{mag}$. The values of dust mass lies between $4.14 \times 10^{34} \mathrm{~kg}$ to $3.37 \times 10^{36} \mathrm{~kg}$ and its mean value found to be $7.98 \times$ $10^{35} \mathrm{~kg}$. Here, we observed the core region of the structure to be less dense than the outer region as a

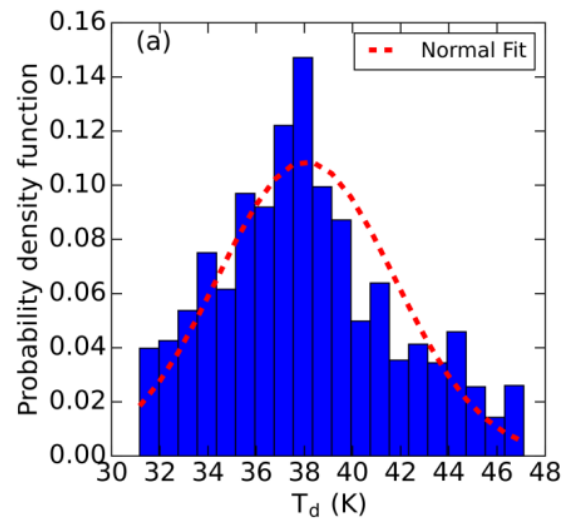

result of the lower extinction in the central part. The mass of dust estimated by Jha et. al, (2017) in cavity is lesser than our calculated dust mass by using AKARI maps at $90 \mu \mathrm{m}$ and $140 \mu \mathrm{m}$ [19].

\subsection{Normal Fit}

Figure 7 (a) and (b) represents the normal distribution plot for dust color temperature and dust mass. In these plots we have tested Gaussian distribution. In figure 7 (a) dust color temperature fitted normal distribution. But in figure 7(b) left skewnes was observed.

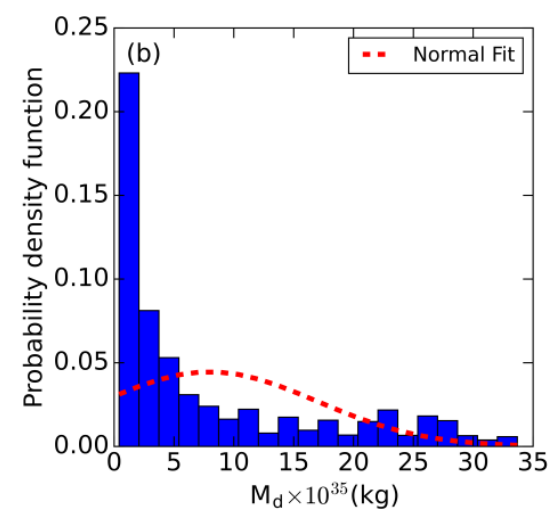

Fig. 7: Normal fit of (a) dust color temperature and (b) dust mass. 
These two plots give the phenomena of inverse trend between dust mass and dust color temperature.

\subsection{Visual Extinction}

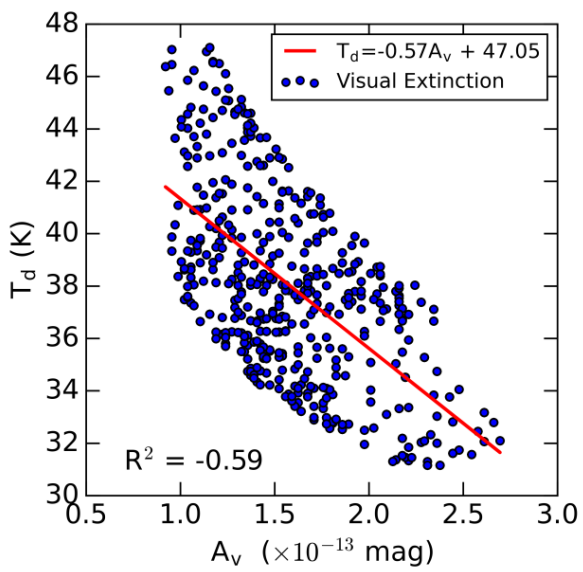

Fig. 8: Correlation between between dust color temperature and visual extinction.

Figure 8 represents the correlation between the dust color temperature and visual extinction around remnant structure. In this case we obtained the best fitted line as:

$T_{\mathrm{d}}=-0.57 A v+47.05$

Here, we obtained the negative correlation between them which yield the correlation coefficient value as $R^{2}=-0.59$. It shows the inverse relation between temperature and extinction.

Figure 9 shows the relation between relative flux density and wavelength at WISE $12 \mu \mathrm{m}, 22 \mu \mathrm{m}$, IRIS $60 \mu \mathrm{m}, 100 \mu \mathrm{m}$ and AKARI $90 \mu \mathrm{m}$ and 140 $\mu \mathrm{m}$. At wavelengths $12 \mu \mathrm{m}$ to $90 \mu \mathrm{m}$ the value of the maximum and minimum relative flux density is found to decrease with an increase in wavelength. But after wavelength $90 \mu \mathrm{m}$ it shows almost constant value of relative flux density.

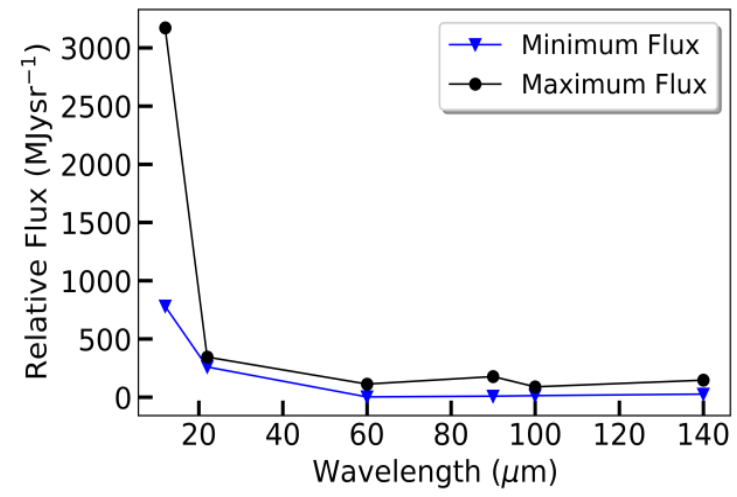

Fig. 9: Relative flux density versus wavelength.

\section{CONCLUSIONS}

We have studied the dust distribution around the supernova remnant using FITS from the Sky View Virtual Observatory of AKARI map with the data reduction software Aladin v11.0 and other supporting programs. We conclude our results as follows:

- The flux density is higher around the middle part of the selected structure. The minimum and maximum flux densities at $90 \mu \mathrm{m}$ are 23.89 $\mathrm{MJysr}^{-1}$ and $177.01 \mathrm{MJysr}^{-1}$ where as at $140 \mu \mathrm{m}$ its value are 55.01 $\mathrm{MJysr}^{-1}$ to $145.94 \mathrm{MJysr}^{-1}$.

- The dust color temperature lies in the range $31.16 \mathrm{~K}$ to $47.11 \mathrm{~K}$ with average value $38.07 \mathrm{~K}$. Similarly values of Planck's function lies between $3.96 \times 10^{-15} \mathrm{Wm}^{-2} \mathrm{sr}^{-1} \mathrm{~Hz}^{-1}$ to $1.96 \times 10^{-14}$ $\mathrm{Wm}^{-2} \mathrm{sr}^{-1} \mathrm{~Hz}^{-1}$ with mean value $9.59 \times 10^{-15} \mathrm{Wm}^{-}$ ${ }^{2} \mathrm{sr}^{-1} \mathrm{~Hz}^{-1}$.

- The value of visual extinction varies from $9.21 \times$ $10^{-14}$ mag to $2.70 \times 10^{-13} \mathrm{mag}$.

- The dust mass lies in the range $4.12 \times 10^{34} \mathrm{~kg}$ and $3.37 \times 10^{36} \mathrm{~kg}$, its mean value found to be $7.98 \times 10^{35} \mathrm{~kg}$ with total dust mass of the nebular structure is found to be $6.11 \times 10^{39} \mathrm{~kg}$ $\left(3.07 \times 10^{9} \mathrm{M}_{\odot}\right)$.

\section{ACKNOWLEDGMENTS}

We are grateful to the anonymous referee for their constructive criticism. We thank the Sky View Virtual Observatory, the AKARI Survey, the SIMBAD database, the Gaia Archive, and Central Department of Physics, Tribhuvan University. One of the authors, DRU acknowledges University Grants Commission of Nepal for SRDI-75/76 S T 11 funds.

\section{REFERENCES}

[1] Bethe, H. A. Supernova mechanisms. Reviews of Modern Physics, 62(4): 801 (1990).

[2] Ferriere, K. M. The interstellar environment of our galaxy. Reviews of Modern Physics, 73(4): 1031 (2001).

[3] Clark, D. H. \& Stephenson, F. R. The historical supernovae. Elsevier (2016).

[4] Kiss, C.; Moór, A. \& Tóth, L. V. Far-infrared loops in the 2nd Galactic Quadrant. Astronomy \& Astrophysics, 418(1): 131-141 (2004).

[5] Könyves, V.; Kiss, C.; Moór, A.; Kiss, Z. T. \& Tóth, L. V. Catalogue of far-infrared loops in the Galaxy. Astronomy \& Astrophysics, 463(3): 1227-1234 (2007). 
[6] Li, A. \& Draine, B. T. Infrared emission from interstellar dust. II. The diffuse interstellar medium. The Astrophysical Journal, 554(2): $\mathbf{7 7 8}$ (2001).

[7] Draine, B. T. Physics of the interstellar and intergalactic medium (Vol. 19). Princeton University Press (2010).

[8] Gall, C.; Hjorth, J.; Watson, D.; Dwek, E.; Maund, J. R.; Fox, O. et al. Rapid formation of large dust grains in the luminous supernova SN 2010j1. Nature, 511(7509): 326-329 (2014).

[9] Kotak, R.; Meikle, W. P. S.; Farrah, D.; Gerardy, C. L.; Foley, R. J.; et al. Dust and the type IIPlateau supernova 2004et. The Astrophysical Journal, 704(1): 306 (2009).

[10] Matsuura, M.; Dwek, E.; Meixner, M.; Otsuka, M.; Babler, B. et al. Herschel detects a massive dust reservoir in supernova 1987A. Science, 333(6047): 1258 (2011).

[11] Cherchneff, I. Dust formation in evolved stars and supernovae: new advances and unsolved problems. Proceedings of the International Astronomical Union, 11(A29B): 166-168 (2015).

[12] Matsuura, M.; Dwek, E.; Barlow, M. J.; Babler, B.; Baes, M. et al. A stubbornly large mass of cold dust in the ejecta of supernova 1987A. The Astrophysical Journal, 800(1): 50 (2015).

[13] Sarangi, A.; Matsuura, M. \& Micelotta, E. R. Dust in supernovae and supernova remnants I: formation scenarios. Space Science Reviews, 214(3): 1-48 (2018).

[14] Draine, B. T. Interstellar dust grains. Annual Review of Astronomy and Astrophysics, 41(1): 241-289 (2003).

[15] Li, A. Spitzer's perspective of polycyclic aromatic hydrocarbons in galaxies. Nature Astronomy, 4(4): 339-351 (2020).

[16] Green, D. A.; Tuffs, R. J. \& Popescu, C. C. Farinfrared and submillimetre observations of the Crab nebula. Monthly Notices of the Royal Astronomical Society, 355(4): 1315-1326 (2004).

[17] Aryal, B.; Rajbahak, C. \& Weinberger, R. A giant dusty bipolar structure around the planetary nebula NGC 1514. Monthly Notices of the Royal Astronomical Society, 402(2): 1307-1312 (2010).

[18] Jha, A. K. \& Aryal, B. A study of pulsar wind driven structure in far-infrared IRAS map at latitude $-10^{\circ}$. Journal of Institute of Science and Technology, 22(1): 1 (2017).

[19] Jha, A. K.; Aryal, B. \& Weinberger, R. A study of dust colour temperature and dust mass distributions of four far-infrared loops. Revista Mexicana de Astronomia y Astrofisica, 53: 467 (2017).

[20] Jha, A. K. \& Aryal, B. A Study of a Cavity Nearby a Pulsar at $-60^{\circ}$ Latitude in the Far
Infrared Map. Journal of Nepal Physical Society, 4(1): 33 (2017).

[21] Jha, A. K. \& Upadhyay, D. R. Dust Structure around two Asymptotic Giant Stars at Latitude $32^{\circ} \& 40.67^{\circ}$. Himalayan Physics, $41-47$ (2017).

[22] Khanal, L.; Upadhyay, D. R.; Jha, A. K. \& Aryal, B. Study of ambient environment around Asymptotic Giant Branch Carbon Star: IRAS 01142+ 6306. BIBECHANA, 16: 31-40 (2019).

[23] Upadhyay, D. R.; Khanal, L.; Hamal, P. \& Aryal, B. Dust Structure Around Asymptotic Giant Branch Stars. Proceedings of the International Astronomical Union, 14(S343): 525-526 (2018).

[24] Jha, A. K. \& Aryal, B. Dust color temperature distribution of two FIR cavities at IRIS and AKARI maps. Journal of Astronomy and Astrophysics, 39(2): 7 (2018).

[25] Joshi, I. N.; Jha, A. K. \& Aryal, B. A study of dust structure nearby white dwarf WD1334678. BIBECHANA, 18(2): 13 (2021).

[26] Upadhyay, D. R. \& Subedi, T. Distribution of dust properties around carbon rich AGB star: IRAS 04427+ 4951 using IRIS and AKARI survey. BIBECHANA, 18(2): 154-163 (2021).

[27] Wood, D. O.; Myers, P. C. \& Daugherty, D. A. IRAS images of nearby dark clouds. The Astrophysical Journal Supplement Series, 95: 457-501 (1994).

[28] Schnee, S. L.; Ridge, N. A.; Goodman, A. A. \& $\mathrm{Li}$, J. G. A complete look at the use of IRAS emission maps to estimate extinction and dust temperature. The Astrophysical Journal, 634(1): 442 (2005).

[29] Dupac, X.; Bernard, J. P.; Boudet, N.; Giard, M.; Lamarre, J. M. et al. Inverse temperature dependence of the dust submillimeter spectral index. Astronomy \& Astrophysics, 404(1): L11L15 (2003).

[30] Hildebrand, R. H. The determination of cloud masses and dust characteristics from submillimetre thermal emission. Quarterly Journal of the Royal Astronomical Society, 24: 267 (1983.

[31] Young, K.; Phillips, T. G. \& Knapp, G. R. Circumstellar shells resolved in IRAS survey data. II-Analysis. The Astrophysical Journal, 409: 725-738 (1993). 